Article

\title{
Extraction of Vertical Walls from Mobile Laser Scanning Data for Solar Potential Assessment
}

\author{
Andreas Jochem ${ }^{1,2, \star}$, Bernhard Höfle ${ }^{3}$ and Martin Rutzinger ${ }^{2,4}$ \\ ${ }^{1}$ alpS-Centre for Climate Change Adaptation Technologies, 6020 Innsbruck, Austria \\ ${ }^{2}$ Institute of Geography, University of Innsbruck, 6020 Innsbruck, Austria; \\ E-Mail: martin.rutzinger@uibk.ac.at \\ ${ }^{3}$ Institute of Geography, University of Heidelberg, 69120 Heidelberg, Germany; \\ E-Mail: bernhard.hoefle@geog.uni-heidelberg.de \\ ${ }^{4}$ ITC Faculty of Geoinformation Science and Earth Observation, University of Twente, 7500 AE \\ Enschede, The Netherlands
}

* Author to whom correspondence should be addressed; E-Mail: andreas.jochem@uibk.ac.at.

Received: 1 February 2011; in revised form: 18 March 2011 / Accepted: 21 March 2011 /

Published: 29 March 2011

\begin{abstract}
In recent years there has been an increasing demand among home owners for cost effective sustainable energy production such as solar energy to provide heating and electricity. A lot of research has focused on the assessment of the incoming solar radiation on roof planes acquired by, e.g., Airborne Laser Scanning (ALS). However, solar panels can also be mounted on building facades in order to increase renewable energy supply. Due to limited reflections of points from vertical walls, ALS data is not suitable to perform solar potential assessment of vertical building facades. This paper focuses on a new method for automatic solar radiation modeling of facades acquired by Mobile Laser Scanning (MLS) and uses the full 3D information of the point cloud for both the extraction of vertical walls covered by the survey and solar potential analysis. Furthermore, a new method is introduced determining the interior and exterior face, respectively, of each detected wall in order to calculate its slope and aspect angles that are of crucial importance for solar potential assessment. Shadowing effects of nearby objects are considered by computing the 3D horizon of each point of a facade segment within the 3D point cloud.
\end{abstract}


Keywords: mobile laser scanning;3D point cloud; 3D feature extraction; solar potential; 3D horizon

\section{Introduction}

With the increasing and unstable prices of fossil fuels and the need to face the serious environmental and economic threats of climate change, renewable energy sources such as wind, biomass and solar energy must play a major role in the energy supply chain. Nowadays, there are many opportunities for households and commercial structures to make use of renewable energy resources and to reduce energy costs, the reliance on fossil fuels and the impact on the environment. This paper focuses on finding suitable places on building facades for the installation of solar panels by performing solar radiation modeling. However, solar radiation modeling cannot be performed without having detailed information about the three dimensional representation of both the buildings of interest and their surrounding objects (e.g., vegetation, infrastructure etc.) that may cause shadowing effects. In recent years Light Detection and Ranging (LiDAR), also referred to as laser scanning has emerged as a standard technology for three dimensional data acquisition. LiDAR is an active optical remote sensing technology that acquires accurate geometrical information of object surfaces by laser range finding. There are two basic types of LiDAR sensors: (i) fixed, ground-based sensors (Terrestrial Laser Scanning, TLS) and (ii) moving sensors that are mounted on mobile platforms such as aircrafts (Airborne Laser Scanning, ALS) or ground vehicles (Mobile Laser Scanning, MLS). Both types of LiDAR sensors store the geometrical information of a scanned surface as a 3D point cloud (XYZ-triples) and each point is tagged with auxiliary information such as timestamp, strength of backscatter and scan angle. Detailed information on LiDAR systems and its applications can be found e.g., in Wehr and Lohr [1] and Vosselman and Mass [2].

The obtained 3D point cloud can be aggregated to $2.5 \mathrm{D}$ raster cells meaning that the surface is represented by a single valued function. This reduces the amount of 3D point cloud data significantly and makes processing less time consuming but leads to an irreversible loss of the third dimension. Hence, 3D shapes such as roof overhangs and caves are not represented in 2.5D raster data. The detection of features such as buildings and facades from laser scanning data can be performed on both 2.5D raster data (e.g., [3-8]) and 3D point cloud data (e.g., [9-13]) and is mostly based on the extraction of planar features. A voxel-based approach extracting planar features from TLS data is presented in Schmitt and Vögtle [14]. Therefore, the original 3D point cloud is converted to a voxel data structure. This reduces the amount of data to process but leads to the loss of the original laser point position due to aggregation of the irregular distributed point cloud into voxel cubes. For each voxel center of gravity the normal vector is calculated. Planar features are derived by grouping adjacent voxels having similar normal vectors. A normal vector based procedure can also be applied to the original 3D point cloud in order to detect planar patches. This requires the inclusion of a point's neighborhood in order to estimate its normal vector [15]. For such a procedure different definitions of neighborhood can be used such as a point's $k$-nearest-neighbors or a point's TIN neighborhood [16-18]. Further information about segmentation techniques is given in Vosselman and Klein [19] and Vosselman et al. [20]. In contrast to a point-wise, 
pixel-wise or voxel-wise classification, a segment-wise classification is more reliable [21] and offers the possibility to assign additional attributes such as slope and aspect to the detected segments. These attributes are of crucial importance when performing solar radiation modeling.

A lot of research has been carried out on the assessment of the incoming solar radiation on roof planes acquired by e.g., ALS [22,23], while only a few have been utilizing the full 3D information of the point cloud for both solar potential assessment and modeling of shadowing effects of nearby objects [16]. Jochem et al. [17] introduced transparent shadow values for vegetation by calculating an averaged local transparency measure for each detected vegetation segment within the 3D point cloud. However, suitable space on roof planes is limited and in some regions roofs are covered in snow for a longer period of time, which might lead to a decrease of energy generation of mounted solar panels. In order to overcome these problems and to increase renewable energy supply solar panels are also mounted on building facades [24-26]. Such panels can be added after the building object has been built or can be integrated with an object's building phase. Facade mounted as well as roof mounted solar panels can be adjusted according to their optimum tilt angle in order to maximize their solar energy collection [27].

Standard ALS data acquisition leads to limited reflections of points from vertical walls caused by (i) large incidence angles, (ii) the relative position of the aircraft to the building wall and (iii) the relatively low point densities. Hence, such data is not suitable to perform a detailed assessment of the incoming solar radiation on building facades. Such operations require the use of TLS and MLS data allowing a more detailed acquisition of building facades. Recent publications in the field of MLS investigate the vertical and horizontal geometric accuracy of MLS measurements [28,29]. So far, only a few publications have been focusing on the extraction of vertical walls from MLS data (e.g., [13]). Rutzinger et al. [30] segment the 3D MLS point cloud into planar regions from which vertical structures are extracted. The segmentation procedure is based on surface growing segmentation with 3D Hough transform for seed detection as described in Vosselman and Klein [19]. Error assessment is performed by comparing the detected facades with building outlines extracted from a digital cadastral map.

The objective of this paper is the development of a new method for automatic solar radiation modeling of building facades acquired by MLS in order to detect areas that might be suitable for the installation of solar panels. The method uses the 3D point cloud directly for both the extraction of vertical walls covered by the survey and solar potential analysis. Furthermore, a new procedure is introduced determining the interior and exterior face, respectively, of each detected wall segment in order to calculate its slope and aspect angles that are the determining factors for solar potential assessment. Shadowing effects of nearby objects are also considered by computing the 3D horizon of each point of a facade segment within the 3D point cloud. GIS-ready information in the form of vector lines of each facade segment will be provided. This allows "normal" GIS users such as urban planners to further process the data without having deeper knowledge about point cloud processing methods.

The paper is structured as follows: in Section 2 the study area and available datasets are presented. The methodology including a detailed description of each step of the workflow is subject of Section 3. In Section 4 the results are presented and discussed followed by a conclusion and outlook on future studies in Section 5. 


\section{Study Area and Dataset}

The study area is a $800 \mathrm{~m}$ long track located in the city of Bonn (Germany) and is characterized by two storey family houses and block buildings surrounded by trees and bushes. Furthermore, the dataset includes temporal objects such as pedestrians as well as moving and parked cars. The ground elevation changes within the area are minor and range from $115 \mathrm{~m}$ to $117 \mathrm{~m}$ above sea level. The street width varies between $20 \mathrm{~m}$ and $50 \mathrm{~m}$. This results in varying distances between the MLS sensor and the buildings.

The MLS data set was acquired by TopScan GmbH using the Optech Inc. Lynx Mobile Mapper that has two 360 degree rotating laser scanners mounted on the back of the vehicle. In order to obtain an optimal visibility of objects and to limit effects of occlusion, the sensors scan in two nearly vertical planes perpendicular to each other meaning that each sensor is mounted with an angle of 45 degrees to the driving direction. Details on the manufacturer specifications can be found in Table 1 and Optech Data Sheet [31].

Each point of the 3D point cloud is located in time (timestamp) and space (XYZ) in an appropriate georeferenced coordinate system. The vehicle position, and thus the sensor positions, are also tagged with a timestamp but are recorded with a much lower frequency (e.g., $2 \mathrm{~Hz}$ ) than the laser points (e.g., $100 \mathrm{kHz})$.

Table 1. Manufacturer specifications of the MLS system used for data collection.

\begin{tabular}{ll}
\hline Sensors characteristics & Optech Inc. LYNX mobile mapper \\
\hline Maximum range & $100 \mathrm{~m}$ (to 20\% reflectivity target) \\
Range precision & $7 \mathrm{~mm}(1$ sigma) \\
Absolute accuracy & $5 \mathrm{~cm}($ at $100 \mathrm{~km} / \mathrm{h})$ (assumes good GPS data) \\
Field of view & $360^{\circ}$ \\
Scan rate & $100 \mathrm{~Hz}$ \\
Measurement rate & 100,000 pulses per second per sensor \\
Echoes per pulse & up to 4 echoes \\
\hline
\end{tabular}

\section{Method}

\subsection{Workflow}

The original MLS point cloud is used as input for segmentation of planar areas. Extraction of building facades and classification of the facades according to their slope and aspect angles (that are of crucial importance for solar potential assessment) are performed within the segmented 3D point cloud containing the detected planar segments. The computation of the incoming solar radiation is executed for each point of each building facade and shadowing effects are computed by calculating the 3D horizon of each point of a facade segment within the 3D point cloud. This requires the use of the original unsegmented MLS point cloud as additional input in order to obtain information about all nearby objects (e.g., vegetation, street lights, buildings etc.) within the study area. By combining the results of the solar radiation model with the calculated area of each segment the total amount of the incoming solar 
radiation per building facade can be computed. In a further step this information is assigned to the 2D lines representing the detected segment. The workflow is shown in Figure 1. Details on each step of the presented workflow are given below.

Figure 1. Workflow for point cloud based solar potential assessment of building facades.

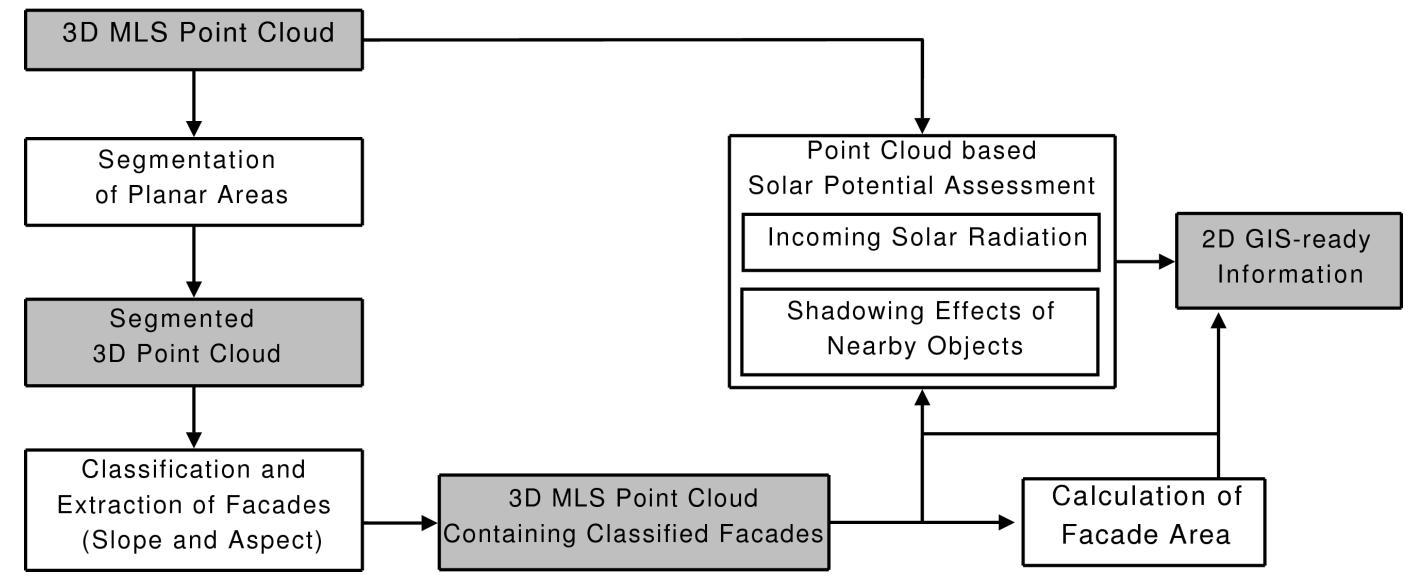

\subsection{Segmentation of Planar Areas}

The laser echoes are grouped to planar segments by surface growing [19], i.e., seed echoes are detected using Hough transform. First a random echo, which is not assigned to a segment yet is selected. Together with a certain number of neighboring echoes these laser echoes are transformed to Hough space. In Hough space those bins are selected, which reach a certain number of votes. These echoes establish the seed surface of the plane. Then plane parameters are estimated by least-squares fitting. Further neighboring echoes are added to the seed surface if they lie in a defined distance to the seed plane. The plane parameters are re-estimated after echoes have been added to the segment. The segment stops growing if no more points are fulfilling the surface growing criteria or the segment size has reached a defined number of points. Points which do not fulfill the surface growing criteria of the current segment are assigned to other segments or remain unsegmented.

\subsection{Classification and Extraction of Building Facades}

Each building facade has two sides. One side faces into the interior of the building while the other side faces to the outside. As the amount of the incoming solar radiation on a building facade is determined by its orientation towards the sun, each detected segment (Section 3.2) is classified according to its slope and aspect angle. This procedure is performed for that side of the building facade facing to the outside. These angles can be calculated by estimating the normal vector of each segment. Therefore, an orthogonal regression plane is fitted to all points of each segment. The normal vector to the fitted plane is considered to be the normal vector of the current segment. As illustrated in Figure 2, the plane fitting procedure can either result in a normal vector pointing into the building or outwards. The normal vector pointing in direction to the corresponding MLS sensor has to be found in order to be able to calculate the slope and aspect angles of the exterior face of the detected segment. The presented procedure requires the timestamp of each point of the 3D point cloud as well as the timestamps of the recorded sensor 
positions. However, the position of the sensor (and its corresponding timestamp) is recorded with a much lower frequency than the laser points (Section 2). The timestamp of a randomly selected point of the current segment is compared to the timestamps of the recorded vehicle positions. The minimum difference between the timestamp values leads to the estimated position of the vehicle at the time the current planar segment was scanned. The appropriate normal vector is determined by calculating the angle between each of the two normal vectors and the vector connecting the randomly selected point and the estimated position of the sensor at the time the detected segment was scanned. The normal vector is considered to point in direction to the vehicle if the calculated angle is smaller than $90^{\circ}$. Once the appropriate normal vector is estimated slope and aspect of the exterior face of each segment can be computed. Slope is defined as the angle between the estimated normal vector and the normal vector with the positive Z-axis of the horizontal plane (XY-plane), i.e., the angle between the horizontal plane and the orthogonal regression plane that is fitted to all points of each segment (Figure 3). Aspect is calculated by projecting the normal vector of the current building facade on the XY-plane and computing the angle (clockwise) from the Y-axis representing the North direction. As the presented study focuses on solar radiation modeling of building facades, only vertical segments having a slope angle of $90^{\circ} \pm 5^{\circ}$ are selected for further analysis. The incoming solar energy on non-vertical segments, unsegmented points as well as on vertical segments that fall below a predefined area size (Section 3.4) is not assessed.

Figure 2. Classification of extracted wall segments according to their slope and aspect angles.

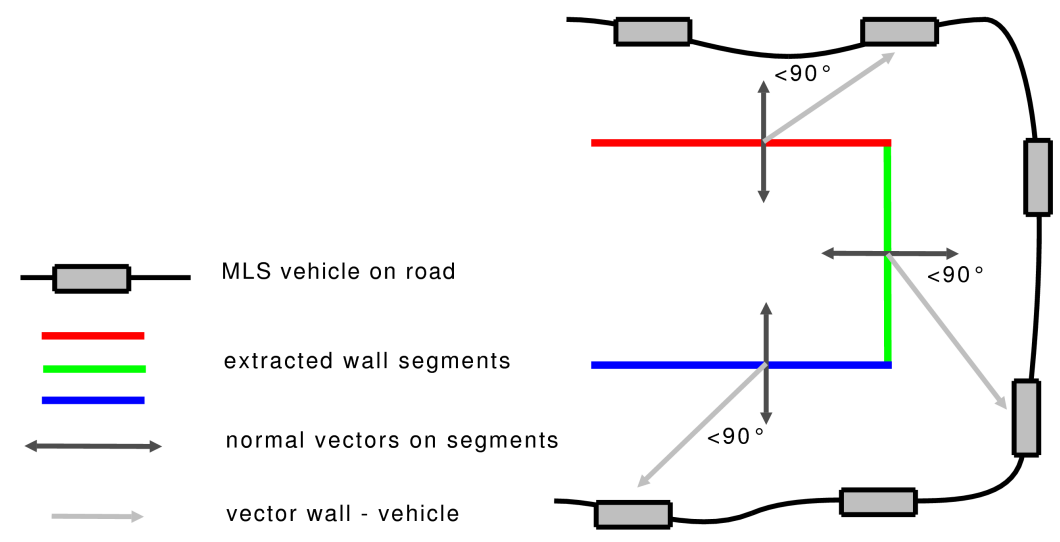

\subsection{GIS-Ready Information of Building Facades}

A vectorization of each detected building object offers the possibility for "normal" GIS users such as urban planners to further process the data without having deeper knowledge about 3D point cloud processing methods in GIS environments. The derived 2D lines can be assigned with additional attributes such as their 3D area and their total amount of incoming solar energy. This allows, for example, a simple differentiation between building objects that might be suitable and unsuitable for the installation of solar panels. By means of 2D alpha shapes [32] the outlines of an unorganized set of 2D data points can be derived and serve as input to calculate the area of each segment [33,34]. This requires a transformation of the point cloud from 3D to $2 \mathrm{D}$ space by maintaining the relative position of the points to each other as illustrated in Figure 3. In a first step an orthogonal regression plane is fitted to all points of each segment. 
After that all points are orthogonally projected on the regression plane before being projected further on the XY-plane. The transformation vector of each point depends on the angle $\beta$ representing the slope of each building facade. After transformation 2D alpha shapes are applied. The optimal alpha value $\alpha$ that is the determining factor of the resulting shape can be estimated from the average point distance. Once the area and the total amount of incoming solar energy per segment is calculated 2D GIS-ready information of each detected building facade segment is produced.

Figure 3. Points being reflected from building facades are projected on the XY-plane in order to apply 2D alpha shapes and calculate the area of each building facade, respectively.

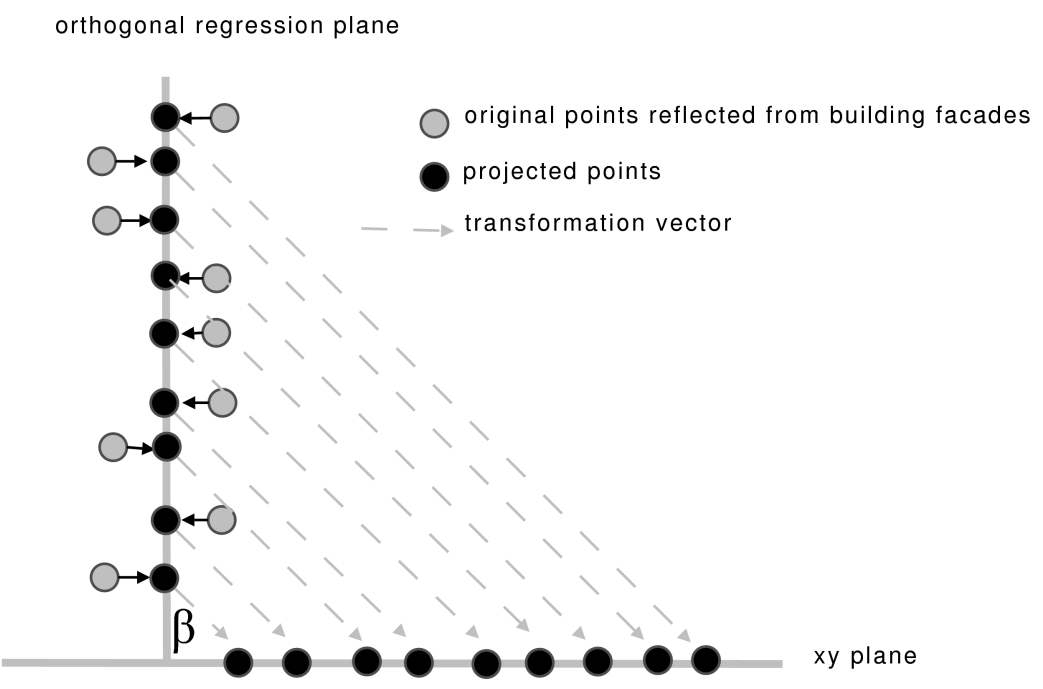

Therefore, the orthogonal regression plane fitted to each building facade segment is intersected with the horizontal XY-plane. The length of the resulting 2D line is equivalent to the length of the corresponding building facade segment (Figure 4).

Figure 4. 2D GIS-ready information in the form of vector lines of each detected building facade segment is generated.

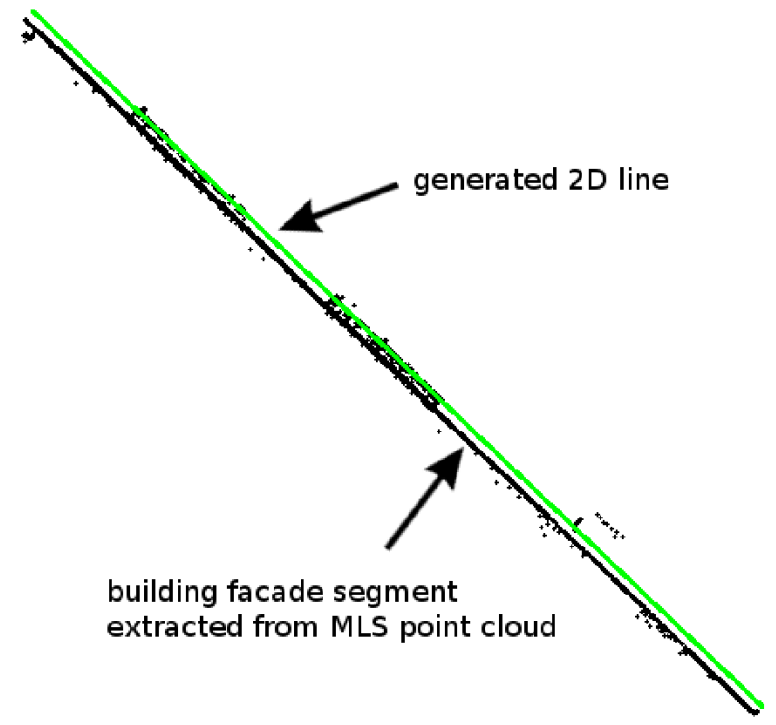




\subsection{Solar Radiation Modeling}

Background

This paper aims at modeling the incoming solar radiation on building facades by using the 3D point cloud of MLS directly, i.e., an aggregation of the 3D point cloud to 2.5D raster cells is not required. The point cloud based solar radiation model was developed in previous studies $[16,17]$ and computes the global incoming solar radiation on each point of the 3D point cloud separately. The global solar radiation is defined as the sum of the direct and diffuse radiation. The direct radiation is defined as the part of the incoming solar radiation reaching the Earth's surface directly without being reflected by the atmosphere. The diffuse radiation is reflected radiation reaching the Earth's surface. Both components are estimated by using formulas presented by Hofierka and Šruri [35]. The position of the sun and its incidence angle at the point of interest as well as sunrise and sunset time are calculated by using the SOLPOS Code that was developed by the National Renewable Energy Library (NREL) [36]. If data from a meteorological ground station close to study area is available, it can be used to consider atmospheric as well as cloud cover effects by calculating the so called clear sky index (CSI) [37] as performed in e.g., Jochem et al. [16] and Jochem et al. [17].

\section{Shadowing Effects}

The presented methodology considers only shadows of nearby objects. Shadows of the surrounding terrain are negligible due to the almost flat topography of the study area. Shadows of nearby objects are assumed to have the greatest influence on the strong local gradients of the incoming solar radiation and are modeled on the highest level of detail. Therefore, the original 3D unsegmented point cloud is used in order to calculate the $3 \mathrm{D}$ horizon of each point being reflected from a building facade within a defined distance (e.g., $40 \mathrm{~m}$ ). This procedure requires both the point cloud containing the classified facades as well as the original MLS point cloud in order to consider all objects possibly casting a shadow. The horizon of each point is computed by determining the minimum solar elevation angle for each azimuth direction (i.e., the angle from the corresponding point to its horizon in direction of the sun). In case the sun lies below the horizon of the corresponding point, the point is considered to be in the shadow of a surrounding object. Furthermore, it is assumed that those points casting a shadow are of a certain size (e.g., $0.3 \mathrm{~m}$ ). This leads to the consequence that points being closer to the current point have a greater influence (i.e., affect a wider range of azimuth angles) than those points being distant. Single points being reflected from, e.g., birds (i.e., points that do not have any neighbors within a defined search distance) are not considered for the calculation of the 3D horizon. Otherwise such points would cast shadows like high objects connected to the ground surface. Linear features such as powerlines can be considered by using methods for linear feature extraction based on eigenvalues in order to determine and exclude those points [38]. Details on the described procedure can be found in Jochem et al. [17].

Solar Potential Assessment

The annual incoming solar radiation is assessed for every single point of the segmented 3D point cloud. It is calculated by the sum of the incoming solar radiation of each day of the year that is computed 
in one hour steps from sunrise to sunset. Shadowing effects of nearby objects are checked by comparing the current elevation angle of the sun with the 3D horizon of the current point. A point is considered to be affected by such shadows if the current 3D horizon (i.e., the minimum required solar elevation angle for the current azimuth direction) is not exceeded by the current elevation angle of the sun. In this case the direct radiation is set to zero and only the diffuse component of the incoming global radiation is computed. This procedure results in the annual solar energy at each point of the segmented 3D point cloud.

\section{Results and Discussion}

\subsection{Segmentation, Classification and Area Calculation}

The segmentation of planar areas is based on Hough transform (Section 3.2) using neighboring points within a search radius of $1.0 \mathrm{~m}$. For seed point selection and growing a distance to plane criterion of $0.2 \mathrm{~m}$ is applied. The bin size in Hough space is set to $3.0^{\circ}$ for the slope angle and $0.2 \mathrm{~m}$ for the distance. This leads to a point cloud containing only planar objects.

Non-planar objects such as vegetation are excluded in large parts (Figure 5). However, if laser points are reflected from very dense vegetation such as from hedges, they are also assumed to be a planar facades. In a further step, non-vertical segments are removed, as they are considered to be roads, curbstones or car roofs. Hence, solely vertical segments having a slope angle of $90^{\circ} \pm 5^{\circ}$ are selected for solar potential assessment. In some cases windows are part of an extracted building facade (indicated by arrows in Figure 6(b)). This could be due to points being reflected from curtains and shutters. A further problem occurs when building facades or parts of building facades are occluded by non-transparent objects such as dense vegetation, walls, cars etc. Hence, no laser points are reflected from the building facade itself. This may lead to both building facades that are incomplete (Figure 6(b)) as well as vertical structures not representing vertical building walls. The latter is the case when objects are also characterized by planarity similar to a building facade such as fences or hedges (Figure 5(b)). As such structures are often small in size (compared to building facades), they can be removed by selecting only those segments exceeding a predefined area size (e.g., $4 \mathrm{~m}^{2}$ ) for further analysis. Small segments representing building facades may also be affected by this procedure. They are considered to be unsuitable for the installation of solar panels. In Figure 5 the results of the segmentation procedure of a subset of the study area are shown and compared to the original MLS point cloud. Points being reflected from roads or curbstone are removed in Figure 5(a) due to visualization reasons. As shown in Figure 5(b) some fences and hedges could not be removed by applying a threshold on area size per segment. They are too large in size. Once the MLS point cloud has been segmented it is used as input for classification of each of the remaining segments according to its slope and aspect angle. The challenge is to find the exterior face (i.e., the side of the facade facing towards the sun) of each segment in order to perform solar radiation modeling properly. In Figure 6(b) the detected facade segments are colored by their aspect angles in order to demonstrate that the exterior facade of all segments is determined. Facades facing in similar directions are characterized by similar aspect angles and are colored the same way. 
Figure 5. The original MLS point cloud (a) is compared to the segmented point cloud (b). The original MLS point cloud is segmented into planar regions, from which vertical structures are extracted. A threshold on area size per segment is applied in order to remove small vertical structures considered to be unsuitable for the installation of solar panels.

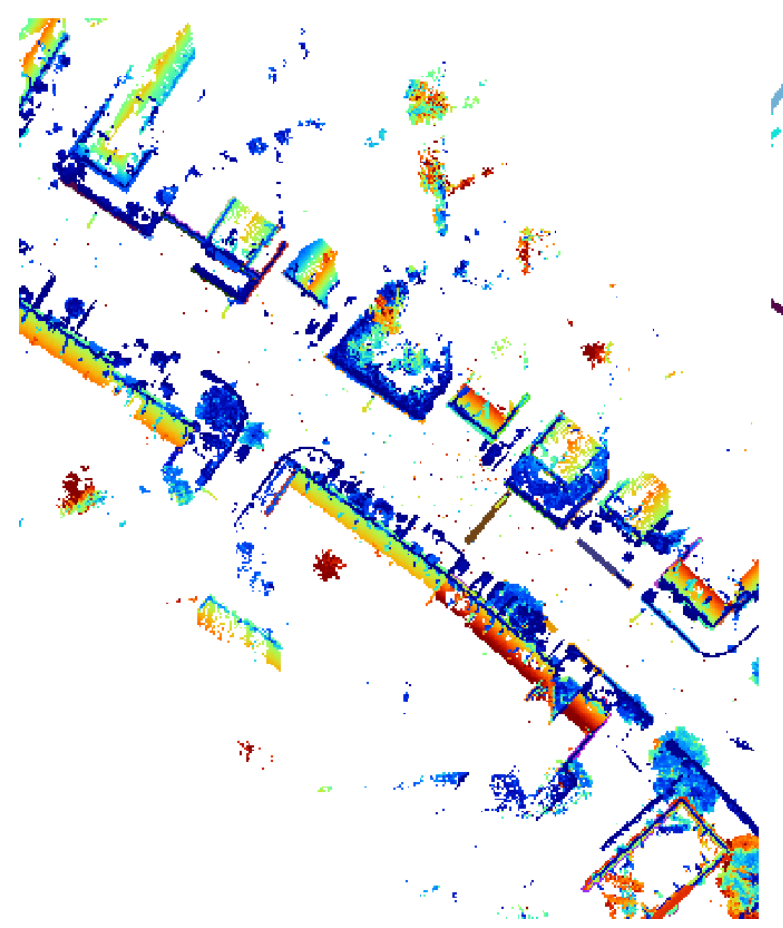

(a) Unsegmented MLS point cloud colored by elevation

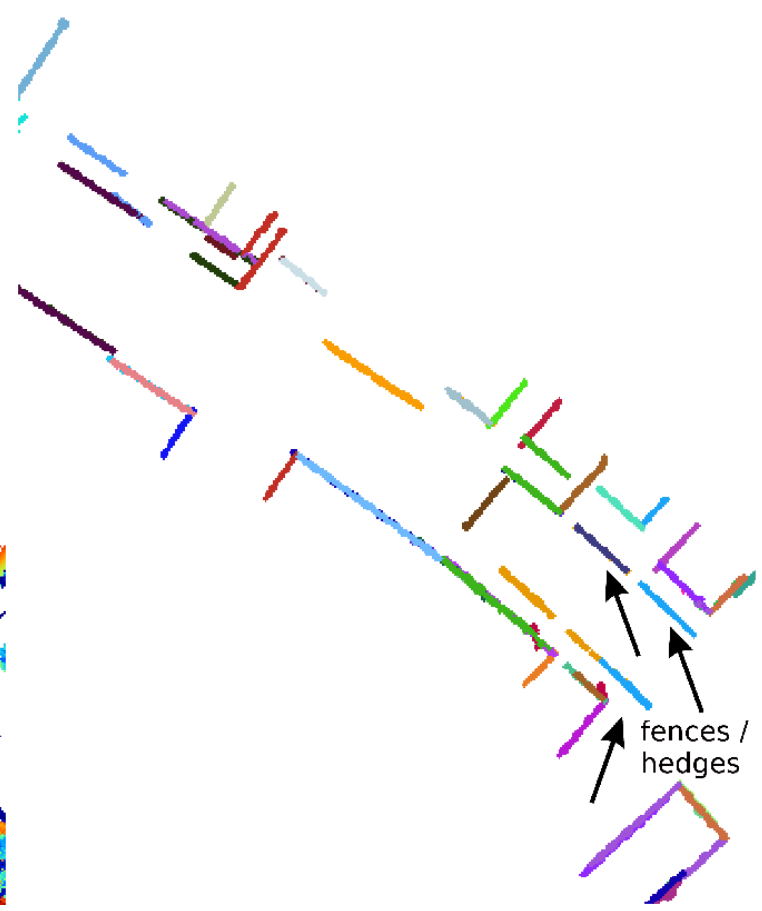

(b) Segmented MLS point cloud colored by segment ID

Figure 6. The derived building facade segments are classified according to their aspect angles. Selected examples are shown in 2D (a) and 3D (b). In some cases segments are incomplete due to occlusion by the nearby objects. Shutters and curtains lead to windows being part of a facade segment.

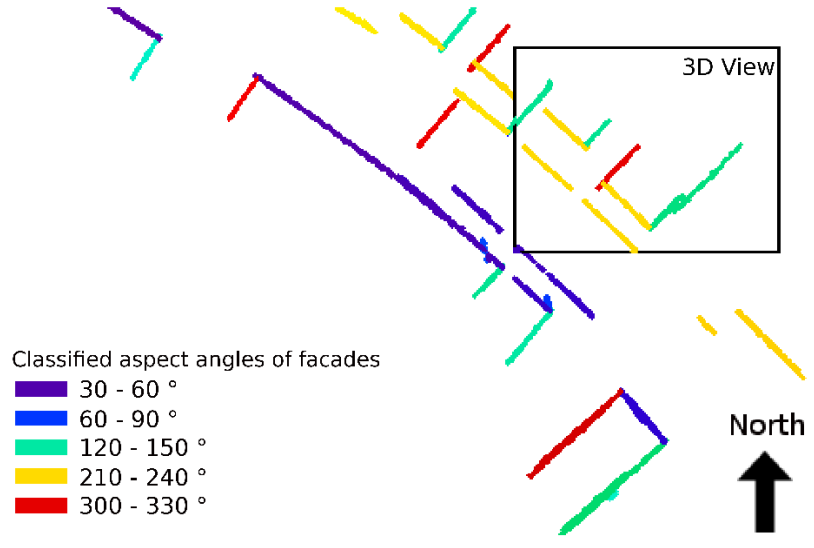

(a) 2D view of aspect classified segments

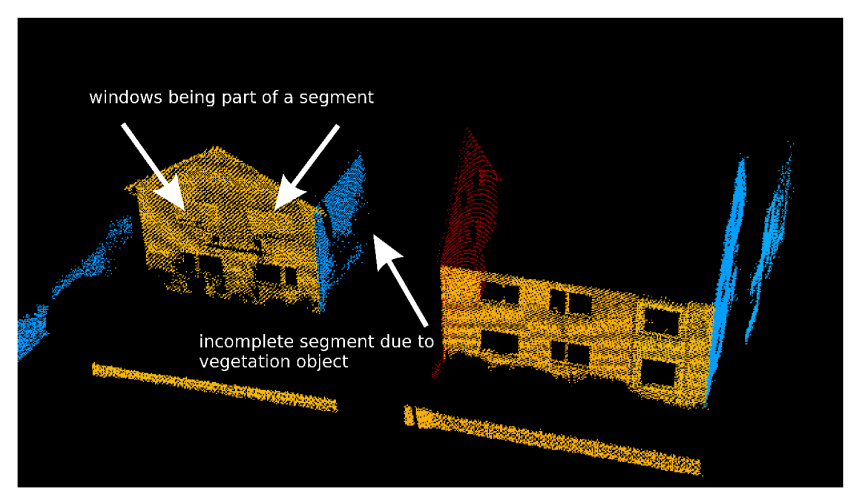

(b) 3D view of aspect classified segments

The used MLS system is only capable of collecting 3D point clouds from building facades along road corridors. Building facades that are not visible to the MLS sensors (e.g., facades at the rear of the 
building) are not acquired by the survey. Hence, the geometrical information is missing and the solar potential of such building facades cannot be analyzed. Rutzinger et al. [30] investigated the completeness of facade representation in MLS data by comparing the detected building facades with the visible outlines extracted from a digital cadastral map. The completeness of the detected segments reaches from 50\% to $74 \%$. In our study appropriate reference data is missing. Hence, completeness and correctness of the extracted building facades cannot be assessed.

The area of each building facade segment is estimated by means of $2 \mathrm{D}$ alpha shapes. As shown in Figure 7 objects that are not part but are located within a facade segment such as windows are excluded for the calculation of its area.

Figure 7. Points belonging to a building facade segment are projected on the XY-plane to calculate its area. Locations that are not part of a segment such as windows are excluded from area calculation.

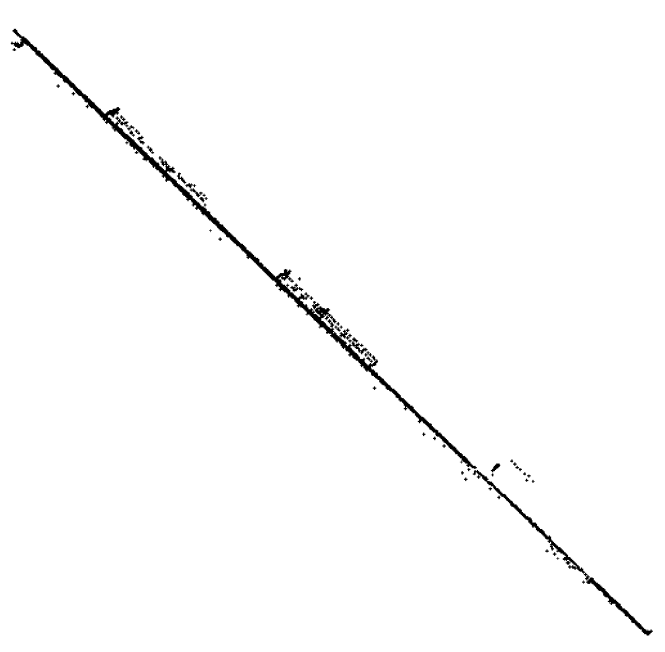

(a) Topview on selected building facade

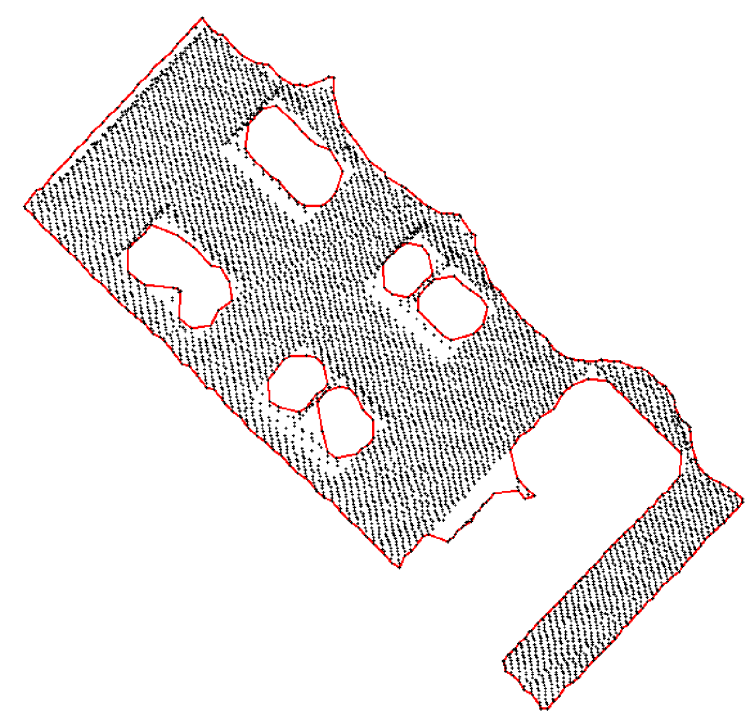

(b) Outline of building facade

\subsection{Solar Radiation Modeling}

The incoming solar radiation on building facades is assessed by using the 3D point cloud for both solar potential assessment and modeling of the 3D horizon of each point of an extracted wall segment. Aggregating the $3 \mathrm{D}$ point cloud to $2.5 \mathrm{D}$ raster cells for shadow mask calculations would speed up processing but suffers from a proper consideration of 3D shapes such as roof overhangs. By performing point cloud based solar radiation modeling the strong local gradients of the insolation are considered in more detail.

The radiation values of the incoming solar radiation model are mainly influenced by the slope and aspect angles of the building facade segments as well as of the search radius that is used to calculate the 3D horizon of each point. Objects that are outside the applied search radius are not considered in the estimated results. The calculation of the 3D horizon is a computationally intensive and very time consuming task. The challenge is to find the optimal search radius in order to include all objects possibly casting a shadow on the corresponding building facade and decreasing computation time to a minimum. 
As the study area is characterized by two storey family houses, the influence of shadowing effects caused by e.g., high rise buildings is not given. Shadows are mainly casted by objects within the corresponding street corridor. As the street width varies between $20 \mathrm{~m}$ and $50 \mathrm{~m}$ (Section 2), the influence of varying search radii $(0 \mathrm{~m}$ to $60 \mathrm{~m})$ on the incoming solar radiation on building facades is analyzed. Therefore, several building facade segments have been selected randomly from the dataset in order to find the optimal search radius for 3D horizon calculations. As shown in Figure 8 the annual incoming solar radiation on the selected building facade segments decreases with increasing search radius. This is due to the consideration of further objects in the estimated results. A search radius of $0 \mathrm{~m}$ indicates that no 3D horizon is calculated. In such cases the incoming solar radiation is overestimated. This underlines the importance of the consideration of shadows of nearby objects. For example building facade segment 6 is overestimated by about $92 \%$. Its mean global solar radiation value decreases from $1,581 \mathrm{kWh} / \mathrm{m}^{2} /$ year when calculating no 3D horizon to $822 \mathrm{kWh} / \mathrm{m}^{2} /$ year when applying a search radius of $60 \mathrm{~m}$ for 3D horizon computations. However, for all selected segments changes in the incoming solar radiation are minor when increasing the search radius from $40 \mathrm{~m}$ to $60 \mathrm{~m}$. As the selected building facades are assumed to be representative for the study area, a search radius of $40 \mathrm{~m}$ is used for the calculation of the 3D horizon of each point being reflected from a building facade.

Figure 8. Investigations to find the optimal search radius for the calculation of the 3D horizon.

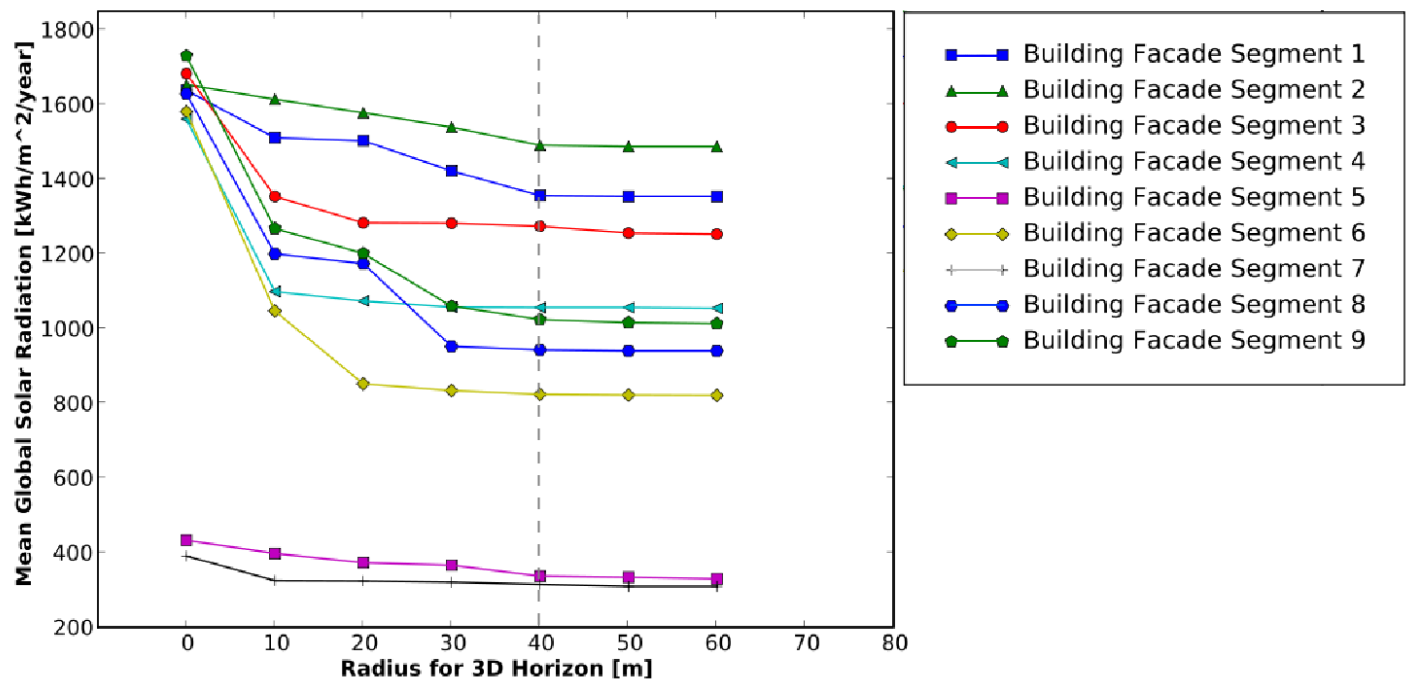

Selected results of the solar radiation model are shown in Figures 9 and 10. Figure 9 shows building facades that are occluded by several vegetation objects casting a shadow, which can be seen by both areas receiving less solar energy and gaps in the segmented point cloud (i.e., incomplete building facade segments). The latter might be due to occluding objects that are nontransparent towards the laser beam. Hence, the whole building facades could not be acquired by the MLS survey. In order to overcome this problem, incomplete facades could be completed by placing virtually generated points having a defined distance to each other within the incomplete parts of a segment, while slope and aspect of the current segment can be assigned to each of the generated points. However, this procedure might also concern windows that are not part of a facade segment. In this study shadows from vegetation objects are treated 
as being shadows of solid objects. Their transparent properties towards the sunlight are not considered as this would require a segmentation of the MLS point cloud into transparent and non-transparent objects as carried out in e.g., Jochem et al. [17]. In order to consider the season dependent shadowing effects of vegetation a classification of vegetation objects into coniferous and deciduous trees is needed to account for leaf-on and leaf-off situations.

Figure 9. The incoming solar radiation is influenced by surrounding objects: (a) Areas being affected by shadowing effects receive less solar energy than areas that are not occluded by any objects. (b) Occluding objects may also lead to incomplete building facades due to their nontransparent properties towards the laser beam.

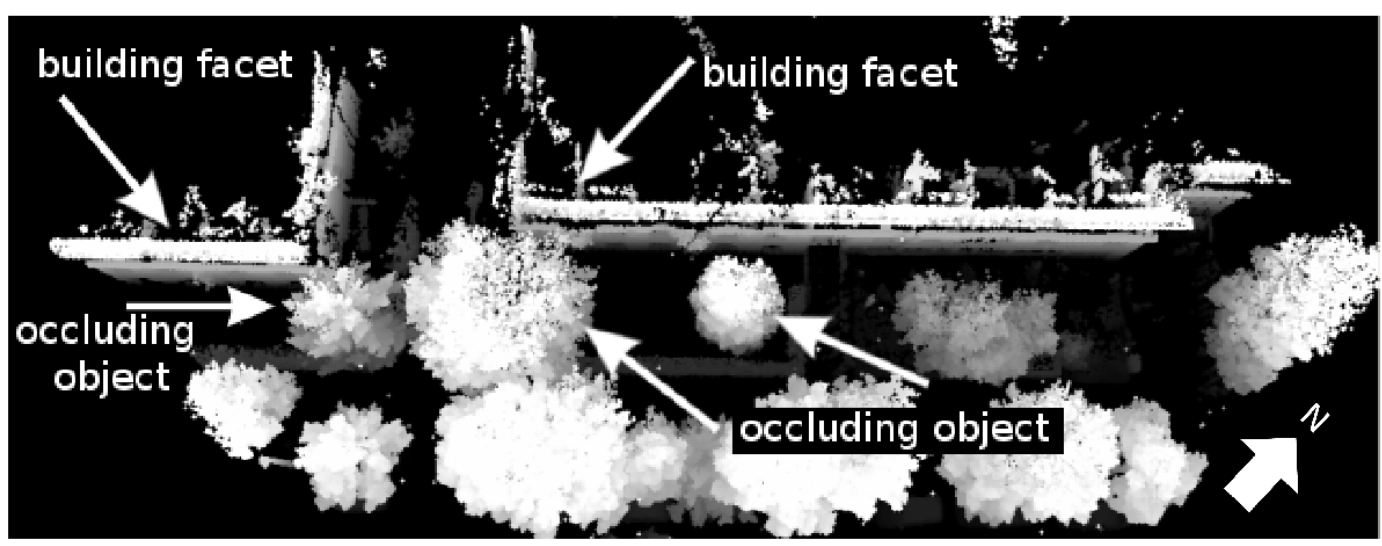

(a) Top view of a subset of the original MLS point cloud showing occluding objects

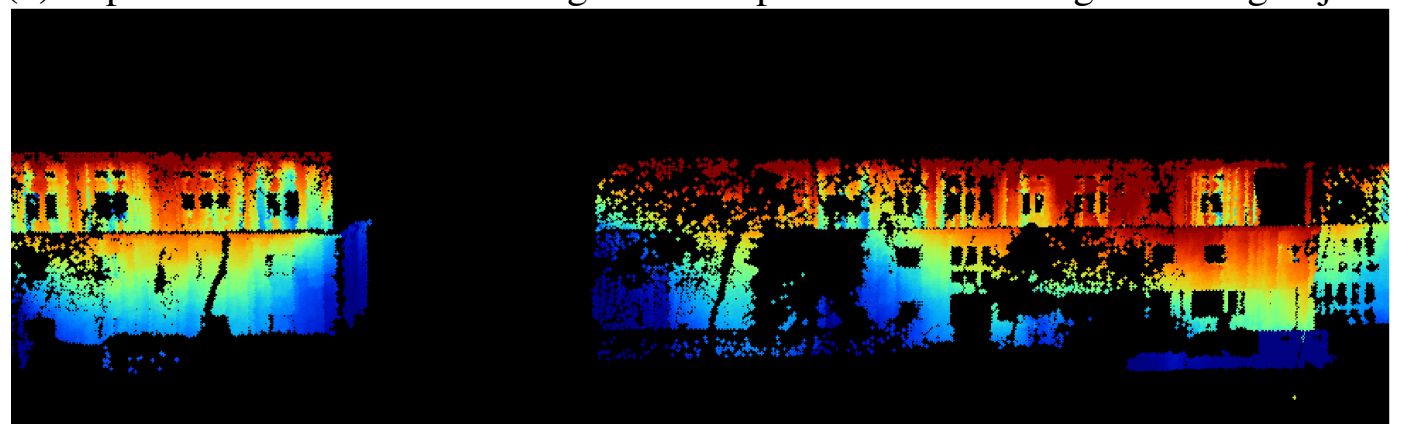

(b) Side view of a subset of the segmented MLS point cloud colored by incoming solar radiation

0 $1700 \mathrm{kWh} / \mathrm{m}^{2} /$ year

Another example of the incoming solar radiation on a building facade is shown in Figure 10. The incoming solar energy on an extracted building facade is calculated by considering shadowing effects of nearby objects. As one can see a nearby tree and a marquee (indicated by arrows in Figure 10(a)) have a strong influence on the incoming solar energy. Areas being affected by their shadows receive less solar energy than areas that are not affected by any shadows. However, the marquee can be seen as a temporal object, which could be removed in order to increase the incoming solar energy on those areas being affected by its shadows. As temporal objects (e.g., pedestrians, moving cars, marquees etc.) are not removed from the MLS dataset, their shadowing effects are also included in the solar radiation model. This might lead to areas not being considered as suitable for the installation of solar panels, although they are only influenced by shadows of temporal objects. Calculating the incoming solar radiation on each building facade without considering shadows of nearby object would result in an equal amount of 
Figure 10. Incoming solar energy on a west southwest orientated building facade is shown. Shadows casted by a nearby tree and a marquee, respectively, result in areas receiving less solar radiation.

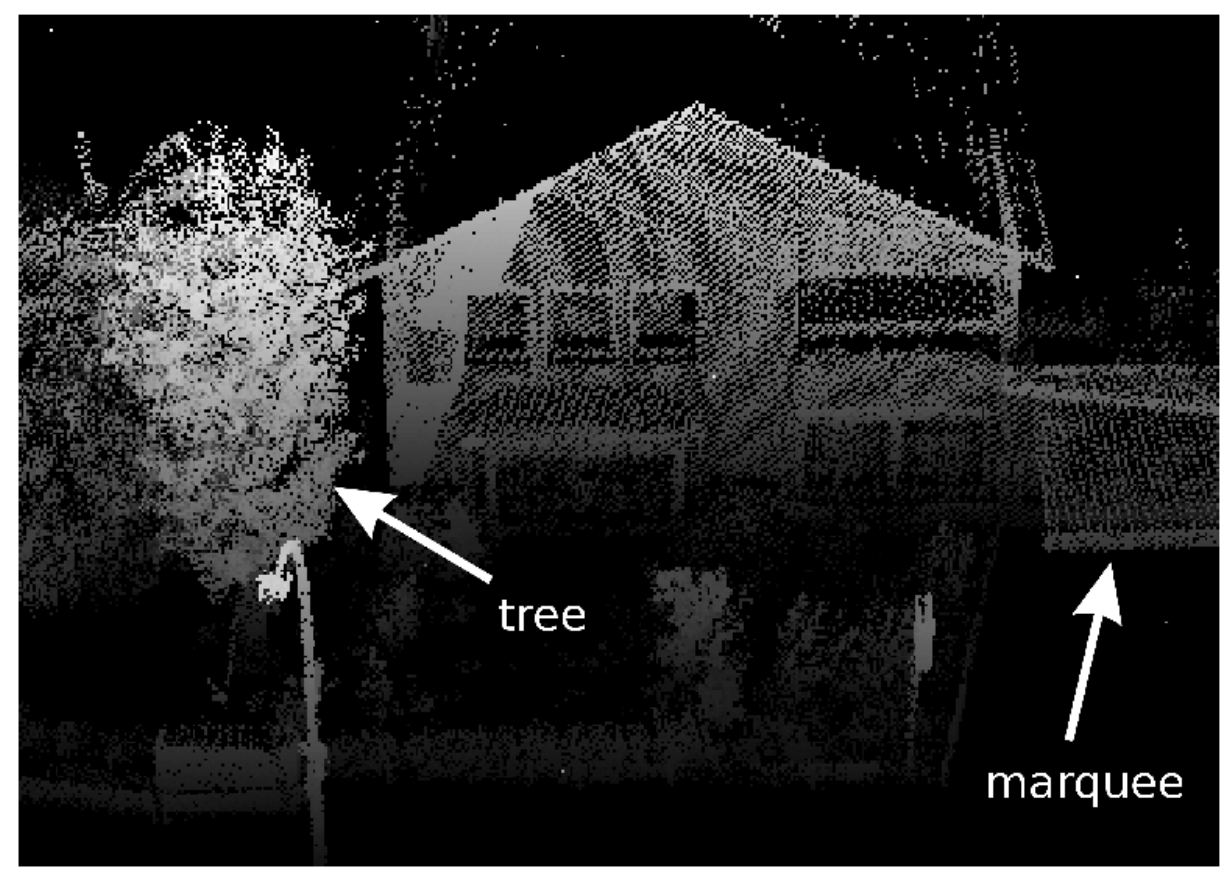

(a) Original MLS point cloud

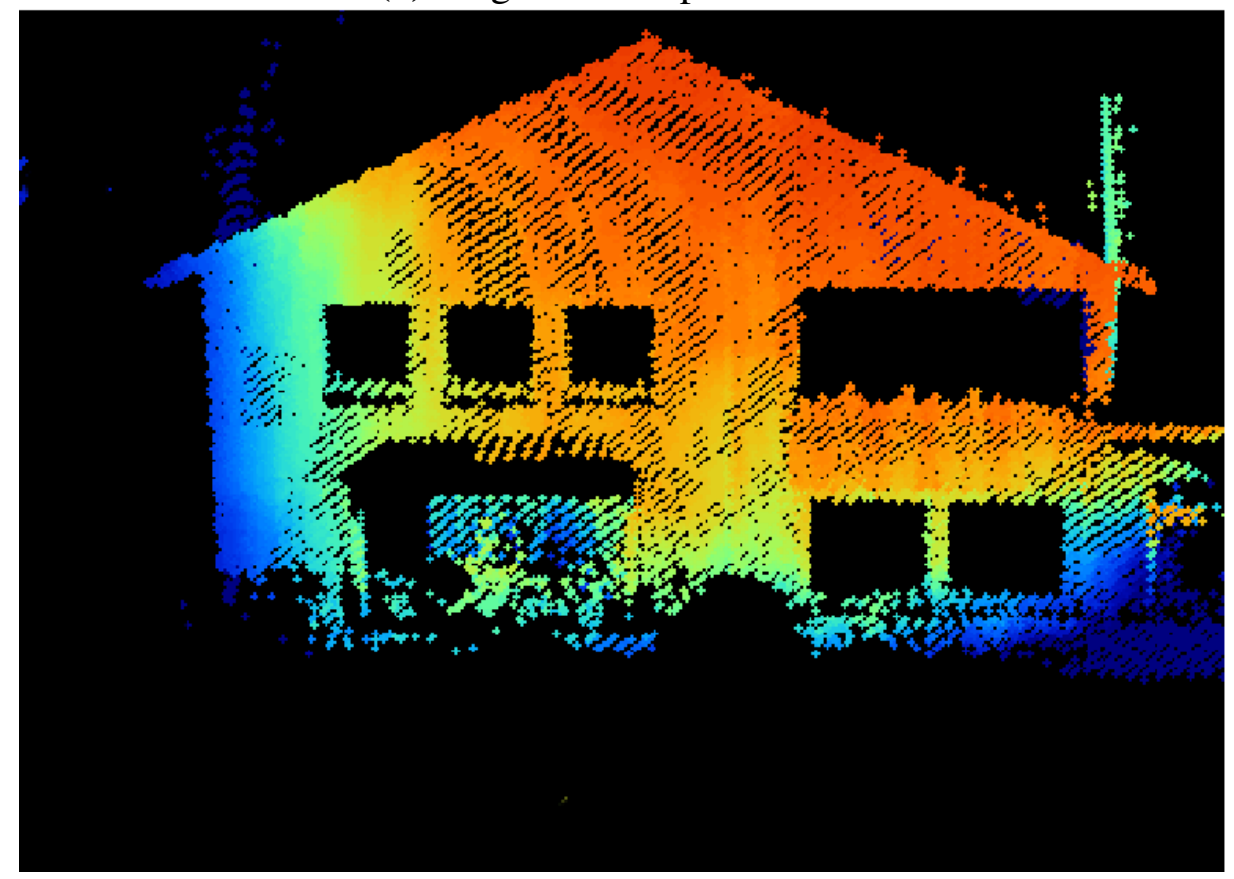

(b) Calculated solar energy on building facade

0 $1700 \mathrm{kWh} / \mathrm{m}^{2} /$ year

solar energy in each part of a facade segment due to equal slope and aspect angles. Due to lack of data from a meteorological ground station close to the study area, the CSI (Section 3.5) is not applied. Hence, a correction of the modeled values and a consideration of atmospheric as well as of cloud cover effects is missing. Nevertheless, the presented method shows high potential to identify locations that are not influenced by shadowing effects and hence might be suitable for the installation of solar panels. 


\section{Summary and Conclusions}

There is an increasing demand among home owners and operators of commercial structures to reduce energy costs, reliance on fossil fuels and the impact on the environment. Therefore, renewable energy has to play a major role in the energy supply chain. Former studies have been investigating the solar potential of roof planes acquired by ALS. Nowadays, solar panels can also be mounted on building facades. In this paper the incoming solar energy on building facades extracted from MLS data is assessed in order to find suitable places for the installation of solar panels and to increase renewable energy supply. The point cloud based solar radiation model uses the full information of the 3D point cloud for both solar potential assessment and modeling of shadowing effects of nearby objects. The latter is considered by computing the 3D horizon of each point of each detected building facade within the 3D point cloud. The extracted building facades are classified according to their slope and aspect angles as these attributes are of determining importance for solar potential assessment. The challenge is to find the exterior face of each segment in order to calculate slope and aspect of the side of the facade facing the outside. The presented procedure requires the timestamp of each point of the 3D point cloud and the timestamp of each recorded vehicle position. As the used MLS system is only capable of collecting 3D point clouds from building facades along road corridors, the geometrical information of building facades that are not visible to the MLS sensors is missing. Hence, solar radiation modeling of such facades cannot be performed in this study. A ground based TLS system could be used to obtain the geometrical information of missing vertical building parts in order to analyze all vertical structures of a building according to the incoming solar energy. The entire 3D information of buildings could also be acquired by using UAVs [39] with laser scanning systems or cameras.

Next steps include the collection of appropriate reference data representing detailed 3D information of building facades in order to develop a method assessing completeness and correctness of the extracted vertical segments. Furthermore, a procedure will be developed completing incomplete building parts that are not visible to the MLS sensor due to occluding objects. The development of methods removing temporary objects such as pedestrians or moving cars that might cast shadows on building facades should also be in the focus of investigation of future research. In this study the modeled values of the incoming solar energy are only approximated absolute numbers due to lack of data from a meteorological ground station close to the study area. Nevertheless, areas on building facades that are not influenced by shadows of nearby objects can be identified by the presented point cloud based solar radiation model. Such areas receive a higher amount of solar energy and might be suitable for the installation of solar panels. However, comprehensive reference data concerning the incoming solar radiation on the detected segments is needed to validate the accuracy and the reliability of the developed model. This is planned to be integrated in the workflow of future studies. Furthermore, the presented method is not limited to point cloud based assessment of the incoming solar energy of building facades. The vectorization (Section 3.4) of the derived segments is useful for spatial planners to further process the data without having knowledge about point cloud processing methods. The derived information can also be integrated in $3 \mathrm{D}$ city models and hedonic price models $[40,41]$. The latter can be used to investigate the willingness of households and homeowners to pay for being exposed to the sun. In summary, the developed methods might contribute to put the outcome of a variety of applications used in spatial science on a higher level. 


\section{Acknowledgments}

The authors would like to thank the TopScan GmbH for granting the use of the MLS data.

\section{References}

1. Wehr, A.; Lohr, U. Airborne laser scanning an introduction and overview. ISPRS J. Photogramm. Remote Sens. 1999, 54, 68-82.

2. Vosselman, G.; Mass, H.G. Airborne and Terrestrial Laser Scanning; Whittles Publishing, CRC Press: Scotland, UK, 2010.

3. Forlani, G.; Nardinocchi, C. Building detection and roof extraction in laser scanning data. In International Archives of the Photogrammetry, Remote Sensing and Spatial Information Sciences; ISPRS: Vienna, Austria, 2001; Volume 34.

4. Matikainen, L.; Hyyppä, J.; Hyyppä, H. Automatic detection of buildings from laserscanner data for map updating. In International Archives of Photogrammetry, Remote Sensing and Spatial Information Sciences; ISPRS: Vienna, Austria, 2003; Volume 34, Part 3/W13, pp. 218-224.

5. Alharthy, A.; Berthel, J. Detailed building reconstruction from airborne laser data using a moving surface method. In International Archives of Photogrammetry, Remote Sensing and Spatial Information Sciences; ISPRS: Vienna, Austria, 2004; Volume 35, Part B3, pp. 213-218.

6. Rottensteiner, F.; Trinder, J.; Clode, S.; Kubik, K. Automated delineation of roof planes from lidar data. In International Archives of Photogrammetry, Remote Sensing and Spatial Information Sciences; ISPRS: Vienna, Austria, 2005; Volume 36, Part 3W/19, pp. 221-226.

7. Rutzinger, M.; Rottensteiner, F.; Pfeifer, N. A comparison of evaluation techniques for building extraction from airborne laser scanning. IEEE J. Sel. Topics Appl. Earth Obs. Remote Sens. 2009, $2,11-20$.

8. Gorte, B. Planar feature extraction in terrestrial laser scans using gradient based range image segmentation. In International Archives of Photogrammetry, Remote Sensing and Spatial Information Sciences; ISPRS: Vienna, Austria, 2007; Volume 36, Part 3/W52, pp. 173-177.

9. Dorninger, P.; Pfeifer, N. A comprehensiv automated 3D approach for building extraction,

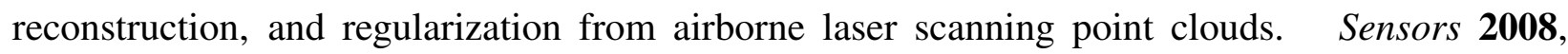
$8,7323-7343$.

10. Rutzinger, M.; Höfle, B.; Pfeifer, N. Object detection in airborne laser scanning data - an integrative approach on object-based image and point cloud analysis. In Object-Based Image Analysis: Spatial Concepts for Knowledge-Driven Remote Sensing Applications; Blaschke, T., Lang, S., Hay, G., Eds.; Springer-Verlag: Berlin/Heidelberg, Germany, 2008; pp. 645-662.

11. Oude Elberink, S.; Vosselman, G. Building reconstruction by target based graph matching on incomplete laser data : Analysis and limitations. Sensors 2009, 9, 6101-6118.

12. Rutzinger, M.; Oude Elberink, S.; Pu, S.; Vosselman, G. Automatic extraction of vertical walls from mobile and airborne laser scanning data. In International Archives of Photogrammetry and Remote Sensing and Spatial Information Sciences; ISPRS: Vienna, Austria, 2009; Volume 38, Part 3/W8, pp. 7-12. 
13. Manandahar, D.; Shibasaki, R. Auto-extraction of urban features from vehicle-borne laser data. In Proceedings of Commission IV Symposium "Geospatial Theory, Processing and Applications", Ottawa, ON, Canada, 9-12 July 2002; Volume 34, Part 4, pp. 433-438.

14. Schmitt, A.; Vögtle, T. An advanced approach for automatic extraction of planar surfaces and their topology from point clouds. Photogrammetrie, Fernerkundung und Geoinformation 2009, $1,43-52$.

15. Filin, S.; Pfeifer, N. Neighborhood systems for airborne laser data. Photogramm. Eng. Remote Sensing 2005, 71, 743-755.

16. Jochem, A.; Höfle, B.; Rutzinger, M.; Pfeifer, N. Automatic roof plane detection and analysis in airborne LiDAR point clouds for solar potential assessment. Sensors 2009, 9, 5241-5262.

17. Jochem, A.; Höfle, B.; Hollaus, M.; Rutzinger, M. Object detection in airborne LiDAR data for improved solar radiation modeling. In International Archives of the Photogrammetry, Remote Sensing and Spatial Information Sciences; ISPRS: Vienna, Austria, 2009; Volume 38, Part 3/W8, pp. 1-6.

18. Rabbani, T.; Heuvel, F.; Vosselman, G. Segmentation of point clouds using smoothness constraints. In International Archives of Photogrammetry and Remote Sensing and Spatial Information Sciences; ISPRS: Vienna, Austria, 2006; Volume 36, pp. 248-253.

19. Vosselman, G.; Klein, R. Visualisation and structuring of point clouds. In Airborne and Terrestrial Laser Scanning; Vosselman, G., Maas, H.-G., Eds.; Whittles Publishing: Scotland, UK, 2010; pp. 43-79.

20. Vosselman, G.; Gorte, B.; Sithole, G.; Rabbani, T. Recognising structure in laser scanning point clouds. In International Archives of Photogrammetry, Remote Sensing and Spatial Information Sciences; ISPRS: Vienna, Austria, 2004; Volume 36, Part 8/W2, pp. 33-38.

21. Vosselman, G. Advanced point cloud processing. In Photogrammetric Week 2009; Fritsch, D., Ed.; University of Stuttgart: Stuttgart, Germany, 2009.

22. Vögtle, T.; Steinle, E.; Tóvári, D. Airborne laserscanning data for determination of suitable areas for photovoltaics. In International Archives of Photogrammetry, Remote Sensing and Spatial Information Sciences; ISPRS: Vienna, Austria, 2005; Volume 36, Part 3/W19, pp. 215-220.

23. Kassner, R.; Koppe, W.; Schüttenberg, T.; Bareth, G. Analysis of the solar potential of roofs by using official lidar data. In International Archives of Photogrammetry, Remote Sensing and Spatial Information Sciences; ISPRS: Vienna, Austria, 2008; Volume 37, Part B4, pp. 399-403.

24. Pagliaro, M.; Ciriminna, R.; Palmisano, G. BIPV: Merging the photovoltaic with the construction industry. Prog. Photovol.: Res. Appl. 2010, 18, 61-72.

25. Marsh, G. BIPV: Innovation puts spotlight on solar. Renewable Energy Focus 2008, 9, 62-67.

26. Benemann, J.; Chehab, O.; Schaar-Gabriel, E. Building-integrated PV modules. Sol. Energ. Mater. Sol. Cell. 2001, 67, 345-354.

27. Yang, H.; Lu, L. The optimum tilt angles and orientations of PV claddings for building-integrated photovoltaic (BIPV) applications. J. Sol. Energ. Eng. 2007, 129, 253-255.

28. Haala, N.; Peter, M.; Cefalu, A.; Kremer, J. Mobile Lidar Mapping For Urban Data Capture. In Proceedings of the 14th International Conference on Virtual Systems and Multimedia, Limassol, Cyprus, 20-25 October 2008; pp. 95-100. 
29. Barber, D.; Mills, J.; Smith-Voysey, S. Geometric validation of a ground-based mobile laser scanning system. ISPRS J. Photogramm. Remote Sens. 2008, 63, 128-141.

30. Rutzinger, M.; Höfle, B.; Oude Elbernik, S.; Vosselman, G. Feasibility of facade extraction from mobile laser scanning data. Photogrammetrie, Fernerkundung und Geoinformation 2011, In review.

31. Optech Data Sheet. Optech Lynx Mobile Mapper Data Sheet; Technical Report; Optech Inc.: Vaughan, ON, Canada, 2008.

32. Edelsbrunner, H.; Mücke, E. Three-dimensional alpha shapes. ACM Trans. Graph. 1994, $13,43-72$.

33. Höfle, B.; Geist, T.; Rutzinger, M.; Pfeifer, N. Glacier surface segmentation using airborne laser scanning point cloud and intensity data. In International Archives of Photogrammetry, Remote Sensing and Spatial Information Sciences; ISPRS: Vienna, Austria, 2007; Volume 36, pp. 195-200.

34. Da, T. 2D Alpha Shapes. In CGAL-3.3 User and Reference Manual; Board, C.E., Ed.; CGAL Open Source Project, 2006.

35. Hofierka, J.; Šruri, M. The Solar Radiation Model for Open Source GIS: Implementation and Applications. In Proceedings of Open Source GIS-GRASS Users Conference 2002, Trento, Italy, 11-13 September 2002.

36. NREL. NREL 2000-SOLPOS Documentation; Technical Report; National Renewable Energy Laboratory, Center for Renewable Energy Resources Renewable Resource Data Center: Golden, CO, USA, 2002.

37. Súri, M.; Hofierka, J. A new GIS-based solar radiation model and its application to photovoltaic assessment. Trans. GIS 2004, 8, 175-190.

38. Pfeifer, N.; Briese, C. Geometrical aspects of airborne laser scanning and terrestrial laser scanning. In International Archives of Photogrammetry, Remote Sensing and Spatial Information Sciences; ISPRS: Vienna, Austria, 2007; Volume 36, pp. 311-319.

39. Eisenbeiss, H. UAV Photogrammetry. Ph.D. Thesis, ETH Zürich, Institute of Geodesy and Photogrammetry, Zürich, Switzerland, 2009.

40. Paterson, R.W.; Boyle, K.J. Out of sight, Out of mind? Using GIS to incorporate visibility in Hedonic Property Value Models. Land Economics 2002, 78, 417-425.

41. Bin, O.; Crawford, T.W.; Kruse, J.B.; Landry, C.E. Viewscapes and flood hazard: Coastal housing market response to amenities and risk. Land Economics 2008, 84, 434-448.

(c) 2011 by the authors; licensee MDPI, Basel, Switzerland. This article is an open access article distributed under the terms and conditions of the Creative Commons Attribution license (http://creativecommons.org/licenses/by/3.0/.) 